\title{
DESIGN OF THE DUAL CONSTELLATION GPS/GALILEO MOBILE DEVICE FOR IMPROVING NAVIGATION OF THE VISUALLY IMPAIRED IN AN URBAN AREA
}

\author{
K. Drypczewski, Ms. C. \\ M. Moszyński, Assoc. Prof. \\ J. Demkowicz, Ph. D. \\ K. Bikonis, Ph. D. \\ A. Stepnowski, Prof. \\ Gdańsk Univeristy of Technology, Poland
}

\begin{abstract}
The article discusses damages of essential tribological associations in crankshaft and piston systems of large power two-stroke engines used as main engines, which take place during transport tasks performed by those ships. Difficulties are named which make preventing those damages impossible, despite the fact that the technical state of engines of this type is identified with the aid of complex diagnostic systems making use of advanced computer technology. It is demonstrated that one of causes of the damages is the lack of research activities oriented on recognising random properties of the loads leading to those damages. A proposal is made for the loads acting at a given time ton tribological associations in crankshaft and piston systems of internal combustion engines used as main engines to be considered as random variables $Q_{t}$. At the same time the loads examined within a given time interval $t_{r} \leq t \leq t_{z}$ would be considered stochastic processes $\{Q(t): t \geq 0\}$. Essential properties of the loads of the abovementioned tribological associations are named and explained by formulating hypotheses which need empirical verification. Interval estimation is proposed for estimating the expected value $E\left(Q_{t}\right)$ of the load $Q_{t}$ acting at time $t$. A relation is indicated between the mechanical load and the thermal load acting on tribological associations in the ship main engine crankshaft and piston system. A suggestion is formulated that a stochastic form of the relation between these types of load is to be searched for, rather than statistic relation, and a proposal is made to measure the intensity (strength) of the stochastic relation using the Czuprow's convergence coefficient.
\end{abstract}

Keywords: dual constellation receiver; GPS; Galileo; Voice Maps; navigation for the blind; visually impaired; personal navigation device; PND

\section{INTRODUCTION}

The main objective of presented research is a feasible improvement of the performance of existing mobile personal navigation devices (PND) for pedestrian navigation in urban areas, in particular for blind and visually impaired persons.

It is well known by users of PND and other GPS-based devices that positioning suffers from (local) significant decreases of accuracy in partially obscured environments like urbanized areas, where buildings (especially high buildings), trees or terrain block large portions of the sky [4].

In such areas, GPS receiver performance is usually deteriorated by the reduced number of currently available satellite signals, as well as by introducing their multipath. That is why the GPS positioning in dense urban areas is unreliable, with poor positioning accuracy, particularly in the cross-street direction if a street is surrounded by high buildings. This problem is crucial, especially in the context of solutions based on GPS receivers dedicated for users with special requirements. An example may be blind and/or visually impaired persons using the low cost PND to support their movement, when high precision positioning is required, especially in the context of emergency situations.

The above-mentioned problem of existing GPS receivers' performance deterioration in partially obscured environments in the context of GPS errors is illustrated in Figure 1.

As is seen in such urban areas, the geometry of the available GPS signals is strongly affected; as several satellites are hidden for the GPS receiver, as the "lines of sight” (LoS) going across the street are much more likely to cross buildings. It affects significantly the ellipsoidal error and, as mentioned above, makes the accuracy worse, especially in the across-the-street 
direction, where tall buildings usually interfere with the direct "line-of-sight" from many satellites at that moment, casting GPS shadows. However, as it will be shown, the proposed combined use even of a not fully operational Galileo system, in addition to GPS, will considerably enhance the direct signal availability, which should result in substantial increases of positioning accuracy and reliability.

Therefore, the proposed approach for reaching formulated technical objectives consists of examining the feasibility of integrating existing mobile PND platforms with the nextgeneration combined open GPS-Galileo receiver module, which provides:

1) increased number of available (visible) satellites (two constellations),

2) general enhancement of direct satellite signal availability,

3) improved availability of satellite signals in high-rise cities,

4) better coverage at high latitudes.

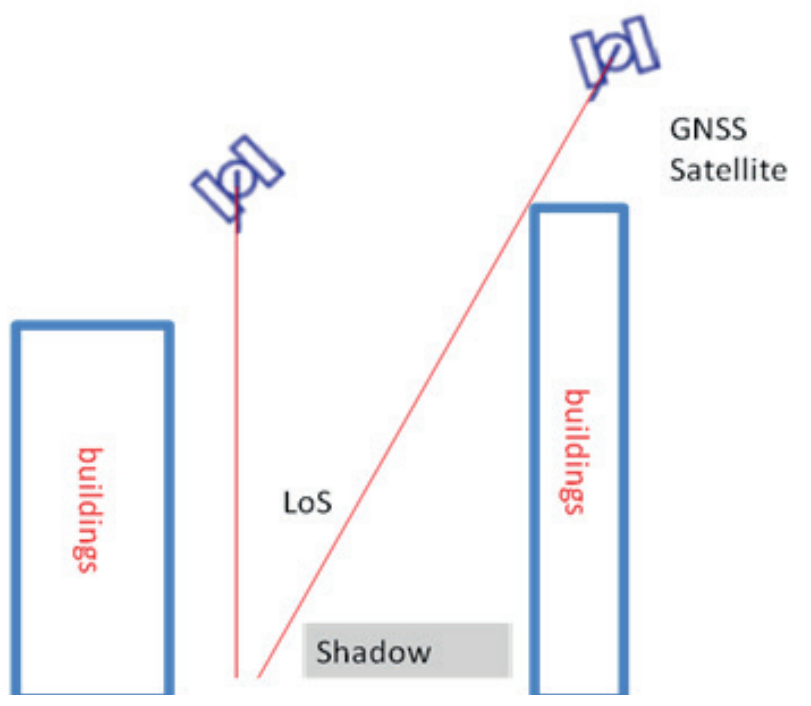

Fig. 1. a) Using direct/non-direct signal reception to determine position

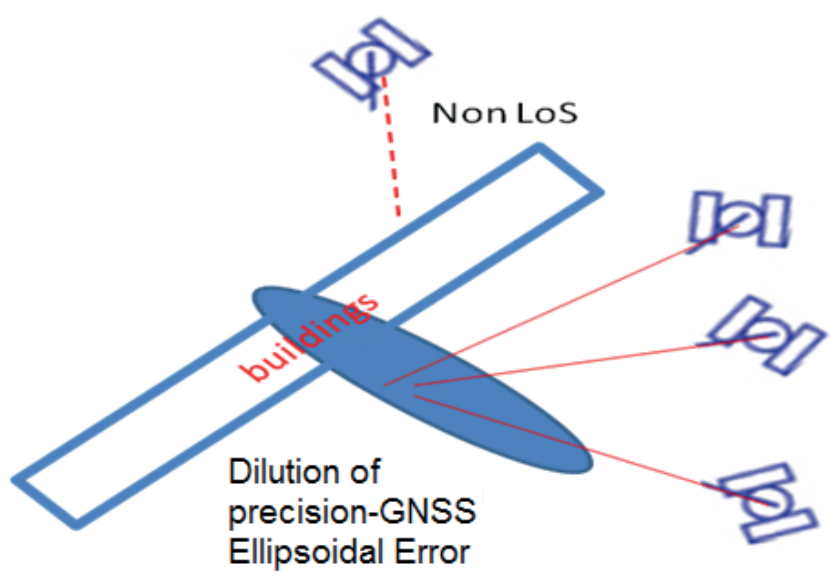

Fig. 1 b) Dilution of precision (DoP) and GPS Ellipsoidal Error - aerial view
These should result in a substantial increase of positioning accuracy and reliability, even for a not fully operational Galileo system

Obviously, future deployment of a fully operational Galileo system, and future broadband signals used in GNSS systems will enhance it further.

In addition to the proposed adoption of the combined GPS/Galileo receiver module in the considered scheme, it is assumed to implement in the project two complementary optional innovations which should further improve positioning accuracy, namely: 1) application of map-matching algorithms, which should eliminate not-possible positioning (users locations) in dense urban areas, e.g. building roofs, 2) application of information source fusion using Bayesian particle filtering algorithms; this seems to be especially promising when $3 \mathrm{D}$ spatial data is available, (e.g. city blocks or building 3D models), and when the filter can be fed with such a priori information.

To summarize, it may be assumed that the fusion of the proposed approaches should provide additional improvements in most environments, not only in highly urbanized areas.

\section{VOICE MAPS}

During the last two years the authors were involved in the team which carried out, and successfully ended, the national project "Voice Maps" in the scheme of the Polish National R\&D Center - NCBR - IniTech Project ZPB/37/67901/IT2/10, [jakaś pozycja literaturowa $\mathrm{z}$ VoiceMaps].

The main goal of that project was to develop a prototype system for the blind and visually impaired users in order to improve their comfort in everyday life by navigating them in the urban areas. The system includes a client application for Android mobile devices and a server $[5,6]$.

The application uses a multimodal user interface; combining mainly voice recognition, dedicated software keyboards, touch-driven menus and speech synthesis mechanisms. The client application calculates the optimal route for the users and then guides them to the selected target along the route. Users' geographical position and their azimuth are continuously monitored, and wrong or dangerous behaviour causes appropriate voice messages to be triggered along with information on passed objects, point of interests (POI) near obstacles and potential dangers, point of danger (POA) [12-14]. Geospatial data is acquired by the community and aggregated on the web portal, allowing preview and editing. The portal currently contains geospatial data of Elbląg city, and partial data for the city of Gdańsk [5, 6].

The "Voice Maps" project solved most of the currently basic problems of pedestrian navigation of the blind in urban terrain; i.e. appropriate digital mapping, navigation algorithms, and timeliness of topography data including the effective spatial data acquisition methods, the efficiency of pathfinding methods, and the appropriate design of the user interface employing voice recognition, speech synthesis mechanisms and touch screens of the modern mobile devices - see Figure 2. 


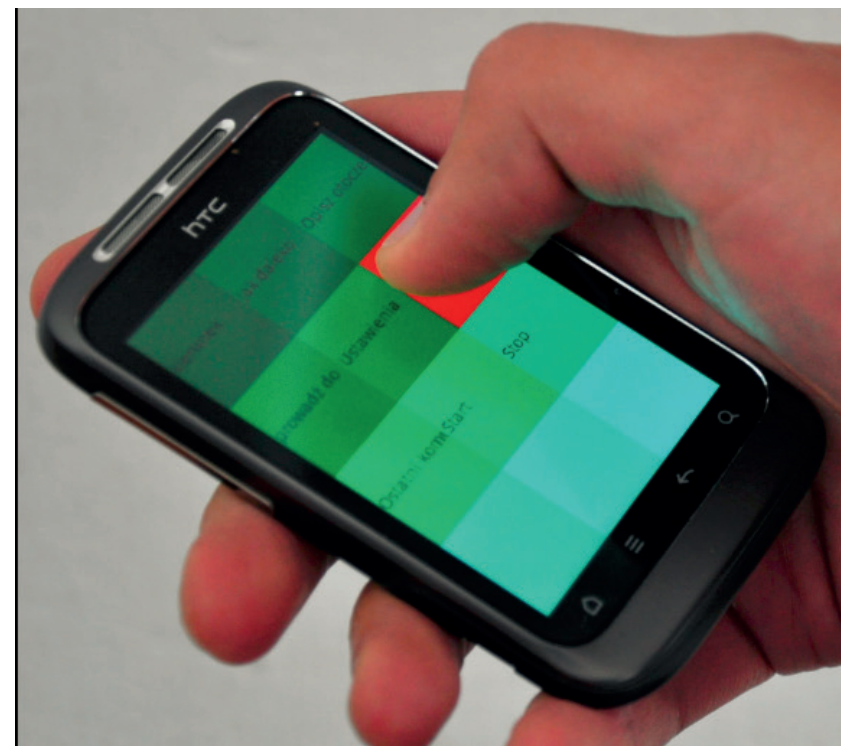

Fig.2. User interface: touch screen menu, map screen for testing, software gesture keyboard

The prototype version of "Voice Maps" system was positively evaluated by the blind consultants and has become the subject of 10 publications and a patent application $[5,6]$.

However, these key problems were solved concurrently with the mobile system development, with one exception which constitutes the insufficient accuracy of positioning in the urbanized areas, which results in the unstable and unreliable performance of the system in difficult urban conditions. Therefore, the achievement of reliable performance of the system, assuring accurate positioning and subsequent navigation of pedestrians, as has become the objective of new research.

\section{MULTICONSTELLATION COMBINED GPS/ GALILEO MOBILE DEVICE}

As was stated in the previous paragraphs, feasibility study of Multiconstellation Combined GPS/Galileo Mobile System consists of two stages: feasibility study itself, and the second stage validating the feasibility of the developed PND system. Study commences with a presentation of the investigation carried out by the "EU-US Cooperation on Satellite Navigation Working Group C", which compares GPS, Galileo and GPS/ Galileo combined performance - simulated for three receivers - and which shows the advantages of the combined GPS/ Galileo system interoperability [2]. Simulations demonstrate and quantify the improvements that can be expected when using GPS and Galileo open services in combination, under different environmental conditions, as shown in Figure 3.

In all cases, the combined approach led to noteworthy performance improvements, as compared to single system performance. The most significant improvement is for urbanized, partially obscured, environments. The increased number of satellites available provides robust performance even when some signals are blocked, which is reflected in a significant increase of positioning accuracy and availability.
As is easily seen, the signals from elevation angles lower than 30 deg are usually discarded for the urban area. This case can be considered as a measure of satellite signal availability in hard urban environments, where direct lines of sight (LoS) below 30deg very often cannot be seen.

The same trends hold true in the $15 \mathrm{deg}$ case, but the overall accuracy is lower here, e.g. $6.45 \mathrm{~m}$ (15 deg) versus $8.02 \mathrm{~m}$ (30 deg) for the SF BOC $(1,1)$ dual constellation case.

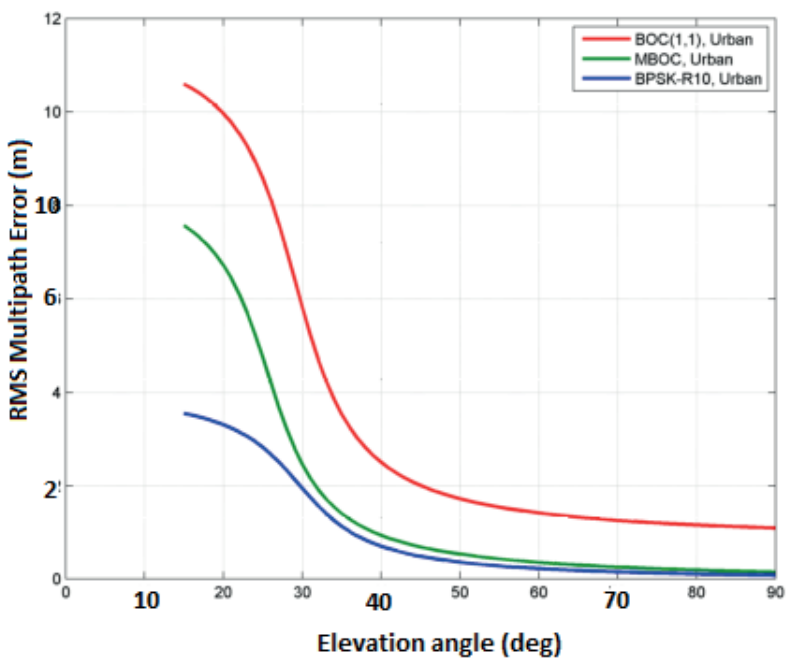

Fig. 3. RMS Multipath Error versus Elevation Angle for single and combined GNSS receivers

Figure 4 presents the user performance for future GPSIII, Galileo, and combined GPS-III/Galileo receivers in different study cases; including open, urban and halfoccluded environments, as well as different ionosphere activity periods.

The figure demonstrates, again, a noteworthy availability improvement due to the combination of both constellations, showing a remarkable improvement in low-visibility areas, as well as deep-urban ones, where non-aviation users are often located when computing their position fix.

Both GPS and Galileo systems, thanks to their interoperable and compatible signal baselines, can be relatively easily integrated and processed, even by civilian-user low-cost equipment. As was shown, due to these reasons, the resulting combined use of the integrated solution offers remarkable benefits to a broad range of user communities.

In the second - development - stage of the approach, the design of the new system integrating existing mobile PND platforms with the next generation, a combined open GPSGalileo receiver module is proposed. This supposes to validate the feasibility of the proposed solution, providing its improved performance (i.e. substantial increase of positioning accuracy and reliability).

The architecture of the system to be developed is presented in the functional block diagram in Figure 5. 


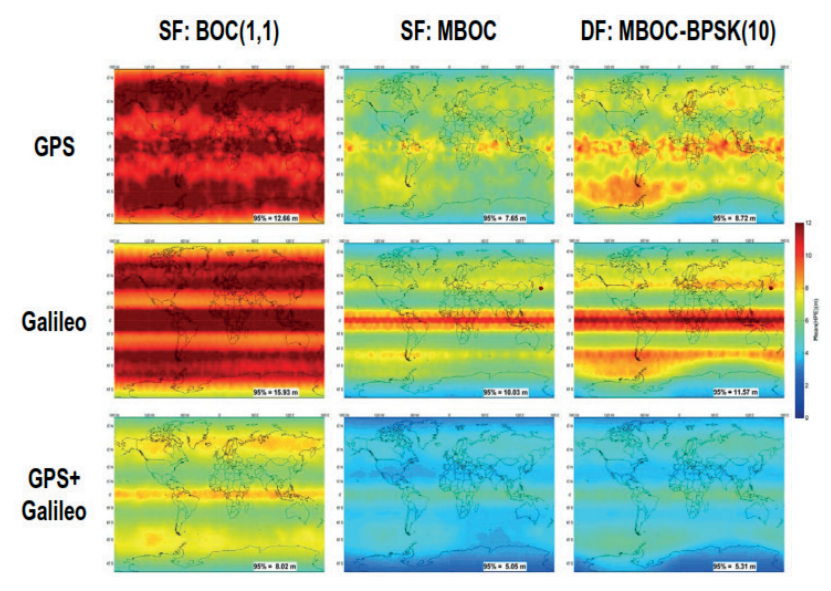

Fig. 4. Urban Global Study (30 deg) - Comparison of Mean Horizontal Position Error HPE $(m)$ for Peak Solar Cycle

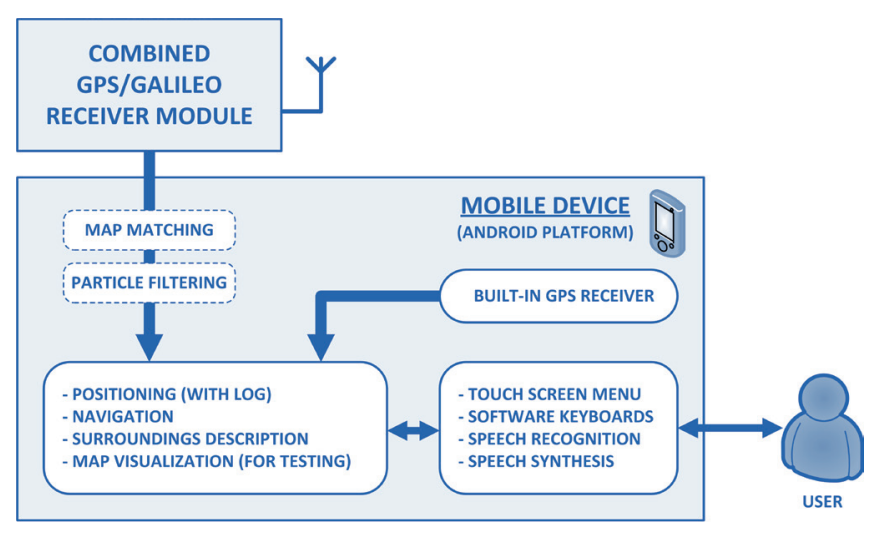

Fig. 5. System Architecture functional block diagram

In addition to the proposed integration of the combined GPS/Galileo receiver module with the existing mobile device smartphone platform - as presented in the scheme - it is assumed to implement in the proposed design two complementary optional software modules, which should further improve positioning accuracy.

The first one refers to implementing map-matching algorithms, which should improve the precision of positioning in urban areas and is described in the next paragraph.

The evaluation of more advanced techniques based on heuristics, fuzzy logic, and the matching of user movements' curves will be also investigated in the next research phase. Correlation and multi-path problems typically lead to high measurement noise and errors. Fuzzy logic and reasoning can additionally filter incorrect measurement from high-noise environments, such as GNSS signals.

Since the history of user movement is known, mathematical analysis of previous measurements can be applied. Polygonal chains of fixed numbers of last positions can be compared with topology of nearest graph vertices to produce more accurate positioning. Moreover, the area surrounded by its intersection with candidate segments can provide additional map-matching parameters.
Submitters are also planning to evaluate if heuristic methods, based on human behaviour and tracking previous movement history, can be included in the map-matching process.

The second one refers to implementing information source fusion using Bayesian particle filtering algorithms.

Particle Filtering is one of the existing methods of sensor information fusion, like Kalman filtering and Extend Kalman Filtering method. PF improves accuracy, especially in situations where there are many sources of information, and where there is a non-linear environment affecting the positioning system i.e. highly urbanized area.

Particle filters work by proposing a hypothesis of where the tracked person is located. Each particle has a position and a weight, and the weight is the confidence we have in that guess. The weight may be treated as the importance of the position calculation (e.g. for the afore-mentioned building roofs it should be reasonably low)

The movement of the particle is restricted by certain rules e.g. some barriers, no walking into walls, or we are walking at constant speed defined in the model, which means that most particles should be moved at that speed. If a particle is far from the reported position from a Galileo receiver, then the particle has its weight lowered, if the particle is close by the reported position it has its weight raised.

So, for highly urbanized areas, this kind of fusion technique seems to be suitable, and therefore seems to very promising. The fusion algorithm is planned to be implemented in the mobile device Android platform.

\section{MAP- MATCHING}

Methods described in previous paragraphs provide solutions to obtain more accurate measurements of the geographical position provided by satellite positioning systems. Even if the acquired position is more reliable, errors connected with time measurement, atmospheric effects and especially multipath issues may still occur. Navigational systems in applications for the blind must be extremely dependable and the need of an additional solution for maximizing positioning quality has emerged.

Map matching is a technique used to determine user geographical position based on location information and electronic maps. In general this problem is difficult because both location and digital geospatial data are typically not accurate $[7,8]$.

Since the representation of the geographical features is kept in the form of the graph, the graph theory definitions will be used. The edge or section in the graph represents linear objects, e.g. streets or sidewalks. The vertices in the graph represent crossroads, points of attention (POA), e.g. stairs, road signs or other obstacles and points of interest (POI), e.g. bus stations or hotels. 
Map matching procedure begins with finding the edges in the area of received geographical location. Search should start from a fixed distance value and if an appropriate number of edges was not found, search should be repeated with higher distance value. Initial value estimation should be based on graph density. Found edges are being referred to as candidate polylines [1] or edges.

In the literature several map matching methods were proposed $[1-3,11]$. The easiest one is based on finding the edge that is closest to the received position. For each of the candidate edges an orthogonal projection of current user position onto the edge is calculated. Distances, which are equal to the length of the segments between determined points and user location, are being compared and the edge with the smallest value is chosen. This solution may produce unpredictable errors as presented in Figure 6. Let us assume that user is moving along the A edge. When he is close to $\mathrm{A}$ and $\mathrm{B}$ segments' intersection his determined position is wrongfully placed on the B edge.

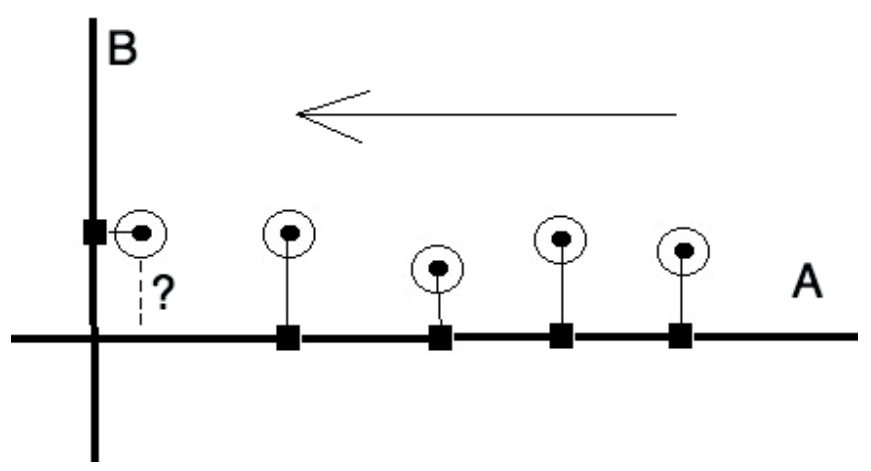

Fig..6. Incorrect map matching to the closest edge

It is clear that another set of parameters must be taken under consideration. Since the information about topological relations in graph and past travel path is available, there is a possibility to use them for error detection. Previously chosen edge can be compared with the new location data to obtain better accuracy. It can be assumed that a user may stay on the last found edge or can move to the next edge when there is a connection between them. Segments that do not have a topological relation with the previous edge should be removed from the candidate edges set. Figure 7 presents situation in which the user would be falsely placed on the B edge instead of A edge if only the closest segment method would be used.

If the time rate of a location data acquisition is high, then the distance travelled between calculated positions is sufficiently short. Therefore other map matching parameters can be established as the length of the path between previous edge and candidate segments. It can be assumed that the edge with the smallest path should be chosen. As shown in Figure 7, the path containing $\mathrm{A}, \mathrm{B}$ and $\mathrm{C}$ edges is longer than the one containing only the A segment.

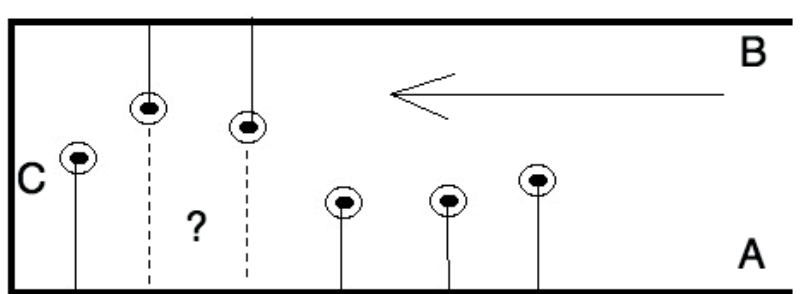

Fig. 7. Incorrect map matching to the edge without topological relation with previously selected edge

Location information contains azimuth of the movement, which can also become a parameter for map matching procedure. The angle of the candidate edge can be compared with the azimuth to estimate the approach degree. It can be assumed that the edge with the smallest angle difference is the one to be chosen during the map matching process. Figure 8 presents a situation in which previously described methods would produce incorrect results. Comparing the angle approach can provide data that will allow determining the correct solution.

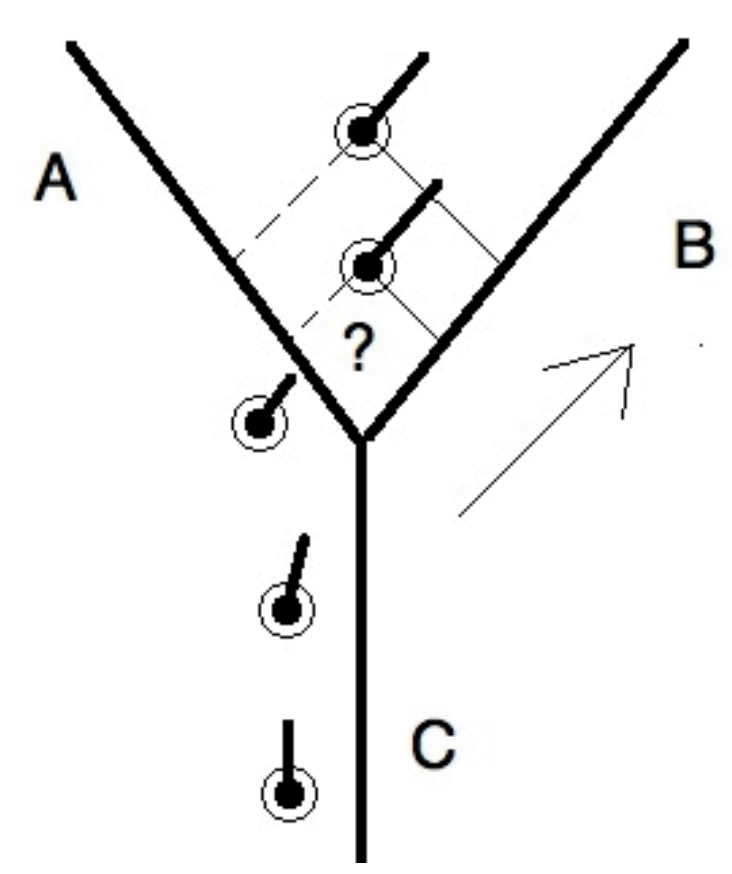

Fig. 8. Map matching using approach degree estimation

The map matching process uses all of the aforementioned methods. The presented algorithm calculates distance $\mathrm{d}$ between received location and the point of the orthogonal projections onto all of the elements of the candidate segments set. Thereafter, the path distance $\mathrm{p}$ as well as the approach degree a is estimated for all edges.

In the last step, results are normalized and the weight function is created. Formula is as follows:

$$
L=\omega_{d} d+\omega_{p} p+\omega_{a} a
$$


where:

$\omega_{\mathrm{d}}$ - distance weight,

$\omega_{\mathrm{p}}$ - path distance weight,

$\omega_{\mathrm{a}}$ - approach degree weight,

Edge with the smallest $L$ value is selected in the implemented map matching process.

\section{SUMMARY}

Contemporary high-end GNSS receivers assisted with referenced systems possess 0.01 meter vertical and horizontal accuracy in open areas, however in dense urbanized areas positioning process is still not reliable. The next-generation combined open GPS-Galileo receiver module, which provides an increased number of available satellites, will provide general enhancement of direct satellite signal availability, therefore improved availability of satellite signals in highrise cities. These should result in a substantial increase of positioning accuracy and reliability, even for a not fully operational Galileo system.

The application of map-matching algorithms, which ought to eliminate not-possible positioning in dense urban areas and application of information source fusion using Bayesian particle filtering algorithms should provide additional improvements in most environments, not only in highly urbanized areas. This is still a far from fully operational receiver concept for the visually impaired, but it is yet another approach toward solving the forward problems which still emerge e.g. sensor integration, integrity etc..

\section{ACKNOWLEDGEMENT}

This research constitutes a sort of followup of the "Voice Maps" project, which was supported by Narodowe Centrum Badań i Rozwoju (National Center of Research and Development) within the "IniTech" program (project code: ZPB /37/679 01/IT2/10).

\section{REFERENCE}

1. L. Xi, Q. Liu, M. Li, Z. Li, Map Matching Algorithm and Its Application, Advances in Intelligent Systems Research, 2007.

2. N. Tradišauskas, D. Tiešyte, C.S. Jensen, A Study of Map Matching for GPS Positioned Mobile Objects, 7th WIM Meeting, 2004.

3. S. Taghipour, M.R. Meybodi, A. Taghipour, An Algorithm for Map Matching For Car Navigation System, Information and Communication Technologies: From Theory to Applications, 1-5, 2008.

4. K. Bikonis, J. Demkowicz, Data Integration from GPS and Inertial Navigation Systems for Pedestrians in Urban Area, International Journal on Marine Navigation and Safety of Sea Transportation, Vol. 7, No. 3, pp. 401-406, 2013.
5. Ł. Kamiński, A. Stepnowski, Ł. Markiewicz, Voice Maps system supporting navigation of the blind, Hydroacoustics, Vol. 15, pp. 83-90, 2012.

6. A. Stepnowski, Ł. Kamiński, J. Demkowicz, Voice Maps - the system for navigation of blind in urban area, Proceedings of the 10th WSEAS international conference on Applied computer and applied computational science, 7-9.03.2011, Venezia, Italy, 2011.

7. L. Yang, Z. Ke, L. Tan, L. Jiabin, The Research On RealTime Map-Matching Algorithm, Proceedings of the International Conference on Industrial Control and Electronics Engineering (ICICEE), Xi'an, China, 2012.

8. Y. Yuan, W. Guan, W. Qiu, Map Matching of Mobile Probes based on Handover Location Technology, Proceedings of the International Conference on Networking, Sensing and Control (ICNSC), Chicago, USA, 2010.

9. J. Meguro, T. Murata, J. Takiguchi, Y. Amano, T. Hashizume, GPS Multipath Mitigation for Urban Area Using Omnidirectional Infrared Camera, IEEE Transactions on Intelligent Transportation Systems, Vol.10, Issue 1, pp. 22-30, 2009.

10. S. Bauer, M. Obst, G. Wanielik, 3D Environment Modeling for GPS Multipath Detection in Urban Areas, Proceedings of the 9th International Multi-Conference on Systems, Signals and Devices (SSD), Chemnitz, Germany, 2010.

11. Wiśniewski B., Drypczewski K., Markiewicz Ł., Kamiński Ł.:Methods of navigation in the mobile application supporting movement of the blind// In: HYDROACOUSTICS.-Vol. 16 (2013),s.243-252

12. Drypczewski K., Kamiński Ł., Markiewicz Ł., Wiśniewski B., Stepnowski A.:Remote Spatial Database Access in the Navigation System for the Blind// In: Marine Navigation and Safety of Sea Transportation: Navigational Problems.-, (2013),s.217-221

13. Markiewicz Ł., Drypczewski K., Wiśniewski B., Kamiński Ł.: Client-server Approach in the Navigation System for the Blind. TransNav, the International Journal on Marine Navigation and Safety of Sea Transportation, Vol. 7 No. 3 ISSN 2083-6473 (e-ISSN 2083-6481).

14. Markiewicz Ł., Drypczewski K., Wiśniewski B., Bieliński T::Application development for Android, iOS and BlackBerry mobile platforms// In Proc. ICT YOUNG 2013/.Gdańsk:Faculty of Electronics, Telecommunications and Informatics, Gdańsk University of Technology,2013,s.51-56

\section{CONTACT WITH AUTHOR}

kszyk@eti.pg.gda.pl 